\title{
Existence and multiplicity of positive solutions to a system of fractional difference equations with parameters
}

\author{
Pimchana Siricharuanun', Saowaluck Chasreechai ${ }^{2}$ and Thanin Sitthiwirattham ${ }^{3^{*}}$ (D)
}

\section{"Correspondence:}

thanin_sit@dusit.ac.th

${ }^{3}$ Mathematics Department, Faculty

of Science and Technology, Suan

Dusit University, Bangkok 10300,

Thailand

Full list of author information is

available at the end of the article

\begin{abstract}
We consider a fractional difference-sum boundary problem for a system of fractional difference equations with parameters. Using the Banach fixed point theorem, we prove the existence and uniqueness of solutions. We also prove the existence of at least one and two solutions by using the Krasnoselskii's fixed point theorem for a cone map. Finally, we give some examples to illustrate our results.
\end{abstract}

MSC: 39A05:39A12

Keywords: Positive solution; System of fractional difference equations; Green's function; Cone

\section{Introduction}

Fractional difference calculus is quite new to researchers. It has been used in mathematical models that explain many real-life situations, for example, economics, electrical networks, and queuing problems (see [1-3] and the references therein). Basic definitions and properties of fractional difference calculus were presented in [4], and discrete fractional boundary value problems have been found in [5-33]. However, the studies of a system of fractional boundary value problems are quite rare (see [34-42]).

For an extension of the research work in this area, in this paper, we study the following system of fractional difference equations with parameters:

$$
\begin{aligned}
& -\Delta^{\alpha_{1}} u_{1}(t)=\lambda_{1} F_{1}\left[t+\alpha_{1}-1, t+\alpha_{2}-1, u_{1}\left(t+\alpha_{1}-1\right), u_{2}\left(t+\alpha_{2}-1\right)\right], \\
& -\Delta^{\alpha_{2}} u_{2}(t)=\lambda_{2} F_{2}\left[t+\alpha_{1}-1, t+\alpha_{2}-1, u_{1}\left(t+\alpha_{1}-1\right), u_{2}\left(t+\alpha_{2}-1\right)\right],
\end{aligned}
$$

subject to nonlocal fractional difference-sum boundary conditions of the form

$$
\begin{aligned}
& \Delta^{-\beta_{1}} u_{1}\left(\alpha_{1}+\beta_{1}-3\right)=\Delta^{\gamma_{1}} u_{1}\left(\alpha_{1}-\gamma_{1}-2\right)=0, \\
& \Delta^{-\beta_{2}} u_{2}\left(\alpha_{2}+\beta_{2}-3\right)=\Delta^{\gamma_{2}} u_{2}\left(\alpha_{2}-\gamma_{2}-2\right)=0, \\
& u_{1}\left(T+\alpha_{1}\right)=\chi_{1} u_{1}\left(\eta_{1}\right), \quad \eta_{1} \in \mathbb{N}_{\alpha_{1}-2, T+\alpha_{1}-1}, \\
& u_{2}\left(T+\alpha_{2}\right)=\chi_{2} u_{2}\left(\eta_{2}\right), \quad \eta_{2} \in \mathbb{N}_{\alpha_{2}-2, T+\alpha_{2}-1},
\end{aligned}
$$

\section{Springer}

(c) The Author(s) 2020. This article is licensed under a Creative Commons Attribution 4.0 International License, which permits use, sharing, adaptation, distribution and reproduction in any medium or format, as long as you give appropriate credit to the original author(s) and the source, provide a link to the Creative Commons licence, and indicate if changes were made. The images or other third party material in this article are included in the article's Creative Commons licence, unless indicated otherwise in a credit line to the material. If material is not included in the article's Creative Commons licence and your intended use is not permitted by statutory regulation or exceeds the permitted use, you will need to obtain permission directly from the copyright holder. To view a copy of this licence, visit http://creativecommons.org/licenses/by/4.0/. 
where $t \in \mathbb{N}_{0, T}:=\{0,1, \ldots, T\}, \alpha_{i} \in(2,3], \beta_{i}, \gamma_{i} \in(0,1), i=1,2$. Moreover, we suppose that the following assumptions hold:

$$
F_{i} \in C\left(\mathbb{N}_{\alpha_{1}-1, T+\alpha_{1}-1} \times \mathbb{N}_{\alpha_{2}-1, T+\alpha_{2}-1} \times(0, \infty) \times(0, \infty),(0, \infty)\right) ;
$$

where $\mathbb{N}_{\alpha_{i}-1, T+\alpha_{i}-1}:=\left\{\alpha_{i}-1, \alpha_{i}, \alpha_{i}+1, \ldots, T+\alpha_{i}-1\right\} ;$

(A2) $0<\chi_{i} \eta_{i}^{\frac{\alpha_{i}-1}{\alpha_{i}}}<\left(T+\alpha_{i}\right)^{\alpha_{i}-1}$;

(A3) $\lambda_{1}, \lambda_{2}$ are positive parameters;

(A4) $\quad F_{i}\left(t_{1}, t_{2}, u_{1}, u_{2}\right)>0$ for $u_{1}, u_{2}>0, t_{i} \in \mathbb{N}_{\alpha_{i}-1, T+\alpha_{i}-1}$.

For convenience, we use the following notations $(i=1,2)$ :

$$
\begin{aligned}
& F_{i}^{0}=\lim _{u_{1}, u_{2} \rightarrow 0^{+}}\left[\max _{t_{i} \in \mathbb{N}_{\alpha_{i}-1, T+\alpha_{i}-1}} \frac{F_{i}\left(t_{1}, t_{2}, u_{1}, u_{2}\right)}{u_{1}+u_{2}}\right], \\
& F_{i}^{\infty}=\lim _{u_{1}, u_{2} \rightarrow \infty}\left[\min _{t_{i} \in \mathbb{N}_{\alpha_{i}-1, T+\alpha_{i}-1}} \frac{F_{i}\left(t_{1}, t_{2}, u_{1}, u_{2}\right)}{u_{1}+u_{2}}\right] .
\end{aligned}
$$

We organize our paper as follows. In Sect. 2, we recall some definitions and basic lemmas and present some properties of the fractional difference operators. In this section, we also derive a representation for the solution to (1.1)-(1.2) by converting the problem to equivalent summation equations. In Sect. 3, we prove the existence and uniqueness result for problem (1.1)-(1.2) by using the Banach fixed point theorem. In Sect. 4, we prove the existence of at least one and two solutions for problem (1.1)-(1.2) by using the Krasnoselskii fixed point theorem in a cone map. In the last section, we provide some examples to illustrate our results.

Theorem 1.1 ([43], Krasnoselskii's fixed point theorem) Let $E$ be a Banach space, and let $K \subset E$ be a cone. Let $\Omega_{1}$ and $\Omega_{2}$ be open subsets of $E$ such that $0 \in \Omega_{1}$ and $\bar{\Omega}_{1} \subset \Omega_{2}$, and let

$$
A: K \cap\left(\bar{\Omega}_{2} \backslash \Omega_{1}\right) \longrightarrow K
$$

be a completely continuous operator such that

(i) $\|A u\| \leq\|u\|, u \in K \cap \partial \Omega_{1}$, and $\|A u\| \geq\|u\|, u \in K \cap \partial \Omega_{2}$, or

(ii) $\|A u\| \geq\|u\|, u \in K \cap \partial \Omega_{1}$, and $\|A u\| \leq\|u\|, u \in K \cap \partial \Omega_{2}$.

Then $A$ has a fixed point in $K \cap\left(\bar{\Omega}_{2} \backslash \Omega_{1}\right)$.

Theorem 1.2 ([44], Arzelá-Ascoli theorem) A set of functions in $C[a, b]$ with the sup norm is relatively compact if and only it is uniformly bounded and equicontinuous on $[a, b]$.

Theorem 1.3 ([44]) If a set is closed and relatively compact, then it is compact.

\section{Preliminaries}

In this section, we provide some notations, definitions, and lemmas, which are used in the main results. 
Definition 2.1 The generalized falling function is defined by $t^{\underline{\alpha}}:=\frac{\Gamma(t+1)}{\Gamma(t+1-\alpha)}$ for any $t$ and $\alpha$ for which the right-hand side is defined. If $t+1-\alpha$ is a pole of the Gamma function and $t+1$ is not a pole, then $t^{\underline{\alpha}}=0$.

Definition 2.2 For $\alpha>0$ and $f$ defined on $\mathbb{N}_{a}$, the $\alpha$-order fractional sum of $f$ is defined by

$$
\Delta^{-\alpha} f(t)=\Delta^{-\alpha} f(t ; a):=\frac{1}{\Gamma(\alpha)} \sum_{s=a}^{t-\alpha}(t-\sigma(s))^{\frac{\alpha-1}{}} f(s),
$$

where $t \in \mathbb{N}_{a+\alpha}$ and $\sigma(s)=s+1$.

Definition 2.3 For $\alpha>0$ and $f$ defined on $\mathbb{N}_{a}$, the $\alpha$ th-order Riemann-Liouville fractional difference of $f$ is defined by

$$
\Delta^{\alpha} f(t):=\Delta^{N} \Delta^{-(N-\alpha)} f(t)=\frac{1}{\Gamma(-\alpha)} \sum_{s=a}^{t+\alpha}(t-\sigma(s))^{\frac{-\alpha-1}{}} f(s),
$$

where $t \in \mathbb{N}_{a+N-\alpha}$, and $N \in \mathbb{N}$ is chosen so that $0 \leq N-1<\alpha \leq N$.

Lemma $2.1([5])$ For $0 \leq N-1<\alpha \leq N$,

$$
\Delta^{-\alpha} \Delta^{\alpha} y(t)=y(t)+C_{1} t^{\frac{\alpha-1}{2}}+C_{2} t^{\frac{\alpha-2}{2}}+\cdots+C_{N} t^{\underline{\alpha-N}}
$$

for some $C_{i} \in \mathbb{R}, 1 \leq i \leq N$.

The following is a solution of a linear variant of the boundary value problem (1.1).

Lemma 2.2 Suppose that $(A 1)-(A 3)$ hold. For $i \in\{1,2\}$, let $\alpha_{i} \in(2,3]$ and $\beta_{i}, \gamma_{i} \in(0,1)$ be given constants, and let $h_{i} \in C\left(\mathbb{N}_{\alpha_{i}-1, T+\alpha_{i}-1}, \mathbb{R}^{+}\right)$be given functions. The problem

$$
\begin{aligned}
& -\Delta^{\alpha_{i}} u_{i}(t)=h_{i}\left(t+\alpha_{i}-1\right), \quad t \in \mathbb{N}_{0, T}, \\
& \Delta^{-\beta_{i}} u_{i}\left(\alpha_{i}+\beta_{i}-3\right)=\Delta^{\gamma_{i}} u_{i}\left(\alpha_{i}-\gamma_{i}-2\right)=0, \\
& u_{i}\left(T+\alpha_{i}\right)=\chi_{i} u_{i}\left(\eta_{i}\right), \eta_{i} \in \mathbb{N}_{\alpha_{i}-2, T+\alpha_{i}-1}
\end{aligned}
$$

has the unique solution

$$
\begin{aligned}
u_{i}\left(t_{i}\right)= & \frac{t_{i}^{\frac{\alpha_{i}-1}{}}}{\left[\left(T+\alpha_{i}\right)^{\frac{\alpha_{i}-1}{2}}-\chi_{i} \eta_{i}^{\frac{\alpha_{i}-1}{i}}\right] \Gamma\left(\alpha_{i}\right)}\left\{\sum_{s=0}^{T}\left(T+\alpha_{i}-\sigma(s)\right)^{\frac{\alpha_{i}-1}{}} h_{i}\left(s+\alpha_{i}-1\right)\right. \\
& \left.-\chi_{i} \sum_{s=0}^{\eta_{i}-\alpha_{i}}\left(\eta_{i}-\sigma(s)\right) \frac{\alpha_{i}-1}{h_{i}}\left(s+\alpha_{i}-1\right)\right\} \\
& -\frac{1}{\Gamma\left(\alpha_{i}\right)} \sum_{s=0}^{t_{i}-\alpha_{i}}\left(t_{i}-\sigma(s)\right)^{\frac{\alpha_{i}-1}{}} h_{i}\left(s+\alpha_{i}-1\right)
\end{aligned}
$$

for $t_{i} \in \mathbb{N}_{\alpha_{i}-3, T+\alpha_{i}}$. 
Proof Using Lemma 2.1 and the fractional sum of order $\alpha_{i} \in(2,3], i \in\{1,2\}$, for (2.1), we obtain

$$
u_{i}\left(t_{i}\right)=C_{1 i} t_{i}^{\alpha_{i}-1}+C_{2 i} t_{i}^{\alpha_{i}-2}+C_{3 i} t_{i}^{\alpha_{i}-3}-\frac{1}{\Gamma\left(\alpha_{i}\right)} \sum_{s=0}^{t_{i}-\alpha_{i}}\left(t_{i}-\sigma(s)\right) \frac{\alpha_{i}-1}{h} h_{i}\left(s+\alpha_{i}-1\right)
$$

for $t_{i} \in \mathbb{N}_{\alpha_{i}-3, T+\alpha_{i}}$.

Next, applying the fractional sum of order $\beta_{i} \in(0,1), i \in\{1,2\}$, to (2.5), we have

$$
\begin{aligned}
\Delta^{-\beta_{i}} u_{i}\left(t_{i}\right)= & \frac{1}{\Gamma\left(\beta_{i}\right)} \sum_{s=\alpha_{i}-3}^{t_{i}-\beta_{i}}\left(t_{i}-\sigma(s)\right)^{\frac{\beta_{i}-1}{2}}\left[C_{1 i} s \frac{\alpha_{i}-1}{2}+C_{2 i} s \frac{\alpha_{i}-2}{2}+C_{3 i} s \frac{\alpha_{i}-3}{}\right] \\
& -\frac{1}{\Gamma\left(\alpha_{i}\right) \Gamma\left(\beta_{i}\right)} \sum_{r=\alpha_{i}}^{t_{i}-\beta_{i}} \sum_{s=0}^{r-\alpha_{i}}\left(t_{i}-\sigma(r)\right)^{\frac{\beta_{i}-1}{}}(r-\sigma(s)) \frac{\alpha_{i}-1}{h_{i}} h_{i}\left(s+\alpha_{i}-1\right)
\end{aligned}
$$

for $t_{i} \in \mathbb{N}_{\alpha_{i}+\beta_{i}-3, T+\alpha_{i}+\beta_{i}}$.

Taking the fractional difference of order $\gamma_{i} \in(0,1), i \in\{1,2\}$, of (2.5), we obtain

$$
\begin{aligned}
\Delta^{\gamma_{i}} u_{i}\left(t_{i}\right)= & \frac{1}{\Gamma\left(-\gamma_{i}\right)} \sum_{s=\alpha_{i}-3}^{t_{i}+\gamma_{i}}\left(t_{i}-\sigma(s)\right) \frac{-\gamma_{i}-1}{2}\left[C_{1 i} s \frac{\alpha_{i}-1}{}+C_{2 i} s \frac{\alpha_{i}-2}{}+C_{3 i} s \frac{\alpha_{i}-3}{}\right] \\
& -\frac{1}{\Gamma\left(\alpha_{i}\right) \Gamma\left(-\gamma_{i}\right)} \sum_{r=\alpha_{i}}^{t_{i}+\gamma_{i}} \sum_{s=0}^{r-\alpha_{i}}\left(t_{i}-\sigma(r)\right)^{\frac{-\gamma-1}{}}(r-\sigma(s))^{\frac{\alpha_{i}-1}{}} h_{i}\left(s+\alpha_{i}-1\right)
\end{aligned}
$$

for $t_{i} \in \mathbb{N}_{\alpha_{i}-\gamma_{i}-2, T+\alpha_{i}-\gamma_{i}}$.

Using the boundary condition $\Delta^{-\beta_{i}} u_{i}\left(\alpha_{i}+\beta_{i}-3\right)=0$ in (2.2), we find that $C_{3 i}=0$.

Then we have

$$
u_{i}\left(t_{i}\right)=C_{1 i} t_{i}^{\alpha_{i}-1}+C_{2 i} t_{i}^{\frac{\alpha_{i}-2}{i}}-\frac{1}{\Gamma\left(\alpha_{i}\right)} \sum_{s=0}^{t_{i}-\alpha_{i}}\left(t_{i}-\sigma(s)\right) \frac{\alpha_{i}-1}{h} h_{i}\left(s+\alpha_{i}-1\right) .
$$

From the boundary condition $\Delta^{\gamma_{i}} u_{i}\left(\alpha_{i}-\gamma_{i}-2\right)=0$ in (2.2) we have $C_{2 i}=0$.

Therefore

$$
u_{i}\left(t_{i}\right)=C_{1 i} t_{i}^{\alpha_{i}-1}-\frac{1}{\Gamma\left(\alpha_{i}\right)} \sum_{s=0}^{t_{i}-\alpha_{i}}\left(t_{i}-\sigma(s)\right)^{\frac{\alpha_{i}-1}{}} h_{i}\left(s+\alpha_{i}-1\right) .
$$

By using the boundary condition (2.3) we obtain

$$
\begin{aligned}
C_{1 i}= & \frac{1}{\left[\left(T+\alpha_{i}\right) \frac{\alpha_{i}-1}{2}-\chi_{i} \eta_{i}^{\frac{\alpha_{i}-1}{i}}\right] \Gamma\left(\alpha_{i}\right)}\left\{\sum_{s=0}^{T}\left(T+\alpha_{i}-\sigma(s)\right)^{\frac{\alpha_{i}-1}{}} h_{i}\left(s+\alpha_{i}-1\right)\right. \\
& \left.-\chi_{i} \sum_{s=0}^{\eta_{i}-\alpha_{i}}\left(\eta_{i}-\sigma(s)\right)^{\frac{\alpha_{i}-1}{}} h_{i}\left(s+\alpha_{i}-1\right)\right\} .
\end{aligned}
$$

Finally, substituting $C_{1 i}$ into (2.9), we obtain (2.4). The proof is complete. 
Corollary 2.1 Problem (2.1)-(2.3) has the unique solution, which is of the form

$$
u_{i}\left(t_{i}\right)=\sum_{s=0}^{T} G_{i}\left(t_{i}, s\right) h_{i}\left(s+\alpha_{i}-1\right)
$$

for $t_{i} \in \mathbb{N}_{\alpha_{i}-3, T+\alpha_{i}}, i=1,2$, where

$$
G_{i}\left(t_{i}, s\right):=\frac{1}{\Gamma\left(\alpha_{i}\right)} \begin{cases}g_{1}\left(t_{i}, s\right), & s \in \mathbb{N}_{0, t_{i}-\alpha_{i}} \cap \mathbb{N}_{0, \eta_{i}-\alpha_{i}}, \\ g_{2}\left(t_{i}, s\right), & s \in \mathbb{N}_{t_{i}-\alpha_{i}+1, \eta_{i}-\alpha_{i}}, \\ 3_{2}\left(t_{i}, s\right), & s \in \mathbb{N}_{\eta_{i}-\alpha_{i}+1, t_{i}-\alpha_{i}}, \\ g_{4}\left(t_{i}, s\right), & s \in \mathbb{N}_{t_{i}-\alpha_{i}+1, T} \cap \mathbb{N}_{\eta_{i}-\alpha_{i}+1, T},\end{cases}
$$

with

$$
\begin{aligned}
& \left.\left.g_{1}\left(t_{i}, s\right):=\frac{t_{i}^{\alpha_{i}-1}}{\left[\left(T+\alpha_{i}\right) \frac{\alpha_{i}-1}{2}-\chi_{i} \eta_{i}^{\alpha_{i}-1}\right.}\right]\left(T+\alpha_{i}-\sigma(s)\right)^{\frac{\alpha_{i}-1}{}}-\chi_{i}\left(\eta_{i}-\sigma(s)\right) \frac{\alpha_{i}-1}{}\right\} \\
& -\left(t_{i}-\sigma(s)\right) \frac{\alpha_{i}-1}{}, \\
& \left.g_{2}\left(t_{i}, s\right):=\frac{t_{i}^{\alpha_{i}-1}}{\left[\left(T+\alpha_{i}\right)^{\frac{\alpha_{i}-1}{2}}-\chi_{i} \eta_{i}^{\alpha_{i}-1}\right.}\right]\left[\left(T+\alpha_{i}-\sigma(s)\right)^{\frac{\alpha_{i}-1}{}}-\chi_{i}\left(\eta_{i}-\sigma(s)\right)^{\frac{\alpha_{i}-1}{}}\right\}, \\
& g_{3}\left(t_{i}, s\right):=\frac{t_{i}^{\alpha_{i}-1}}{\left[\left(T+\alpha_{i}\right) \frac{\alpha_{i}-1}{2}-\chi_{i} \eta_{i}^{\frac{\alpha_{i}-1}{i}}\right]}\left(T+\alpha_{i}-\sigma(s)\right) \frac{\alpha_{i}-1}{}-\left(t_{i}-\sigma(s)\right) \frac{\alpha_{i}-1}{}, \\
& g_{4}\left(t_{i}, s\right):=\frac{t_{i}^{\alpha_{i}-1}}{\left[\left(T+\alpha_{i}\right) \frac{\alpha_{i}-1}{2}-\chi_{i} \eta_{i}^{\frac{\alpha_{i}-1}{i}}\right]}\left(T+\alpha_{i}-\sigma(s)\right) \frac{\alpha_{i}-1}{} .
\end{aligned}
$$

Green's function (2.12) has the following properties.

Proposition 2.1 ([13]) For $i=1,2$, let $G_{i}\left(t_{i}, s\right)$ be Green's function given in (2.12)-(2.13). Then for all $\left(t_{i}, s\right) \in \mathbb{N}_{\alpha_{i}-3, T+\alpha_{i}} \times \mathbb{N}_{0, T}$,

$$
G_{i}\left(t_{i}, s\right) \geq 0
$$

Proposition 2.2 ([13]) For $i=1,2$, let $G_{i}\left(t_{i}, s\right)$ be Green's function given in (2.12)-(2.13). Suppose that for given $\eta_{i} \in \mathbb{N}_{\alpha_{i}-2, T+\alpha_{i}-1}$ and $\alpha_{i} \in(2,3]$, $\chi_{i}$ satisfies the inequality

$$
0 \leq \chi_{i} \leq \min _{\left(t_{i}, s\right) \in \mathbb{N}_{\alpha_{i}-3, T+\alpha_{i}} \times \mathbb{N}_{0, T}}\left\{\frac{\left(T+\alpha_{i}\right) \frac{\alpha_{i}-1}{\alpha_{i}-1}}{\eta_{i}^{\frac{\alpha_{i}}{2}}}-\frac{t_{i}^{\frac{\alpha_{i}-2}{}}\left(T+\alpha_{i}-\sigma(s)\right)^{\alpha_{i}-1}}{\eta_{i}^{\frac{\alpha_{i}-1}{2}}\left(t_{i}-\sigma(s)\right)^{\frac{\alpha_{i}-2}{}}}\right\}
$$

Then

$$
\max _{\left(t_{i}, s\right) \in \mathbb{N}_{\alpha_{i}-3, T+\alpha_{i}} \times \mathbb{N}_{0, T}} G_{i}\left(t_{i}, s\right)=G_{i}(s+\alpha-1, s) .
$$


Proposition 2.3 ([13]) For $i=1,2$, let $G_{i}\left(t_{i}, s\right)$ be Green's function given in (2.12)-(2.13). Then

$$
\min _{t_{i} \in\left[\frac{1}{4}\left(T+\alpha_{i}\right), \frac{3}{4}\left(T+\alpha_{i}\right)\right]} G_{i}\left(t_{i}, s\right) \geq \theta_{i} \max _{\left(t_{i}, s\right) \in \mathbb{N}_{\alpha_{i}-3, T+\alpha_{i}} \times \mathbb{N}_{0, T}} G_{i}\left(t_{i}, s\right)=\theta_{i} G_{i}(s+\alpha-1, s),
$$

where

$$
\begin{aligned}
\theta_{i}:= & \min \left\{\frac{\left(\frac{1}{4}\left(T+\alpha_{i}\right)\right) \frac{\alpha_{i}-1}{\left(T+\alpha_{i}\right) \frac{\alpha_{i}-1}{}}}{}\right. \\
& \left.\frac{1}{\left(\frac{3}{4}\left(T+\alpha_{i}\right)\right) \frac{\alpha_{i}-1}{4}}\left[\left(\frac{3}{4}\left(T+\alpha_{i}\right)\right)^{\frac{\alpha_{i}-1}{2}}-\frac{\left(\frac{3}{4}\left(T+\alpha_{i}\right)\right) \frac{\alpha_{i}-1}{\left(T+\alpha_{i}\right) \frac{\alpha_{i}-1}{}}}{\left(T+\alpha_{i}\right) \frac{\alpha_{i}-1}{}}\right]\right\},
\end{aligned}
$$

and $\theta_{i}$ satisfy the inequality $0<\theta_{i}<1$.

\section{Existence and uniqueness of solution}

In this section, we apply the Banach fixed point theorem to prove the existence and uniqueness result for problem (1.1)-(1.2). For each $i, j \in\{1,2\}$, we let $E_{i}=C\left(\mathbb{N}_{\alpha_{i}-3, T+\alpha_{i}}, \mathbb{R}\right)$ be the Banach space for all functions on $\mathbb{N}_{\alpha_{i}-3, T+\alpha_{i}}$ with the norm $\left\|u_{i}\right\|=\max _{t_{i} \in \mathbb{N}_{\alpha_{i}-3, T+\alpha_{i}}}\left|u_{i}\left(t_{i}\right)\right|$. The product space $\mathcal{U}=E_{1} \times E_{2}$ is a Banach space with the norm

$$
\left\|\left(u_{1}, u_{2}\right)\right\|_{\mathcal{U}}=\left\|u_{1}\right\|+\left\|u_{2}\right\|
$$

Next, define the operator $\mathcal{T}: \mathcal{U} \rightarrow \mathcal{U}$ by

$$
\left(\mathcal{T}\left(u_{1}, u_{2}\right)\right)\left(t_{1}, t_{2}\right):=\left(\left(\mathcal{T}_{1}\left(u_{1}, u_{2}\right)\right)\left(t_{1}, t_{2}\right),\left(\mathcal{T}_{2}\left(u_{1}, u_{2}\right)\right)\left(t_{1}, t_{2}\right)\right)
$$

and for $i, j \in\{1,2\}, i \neq j$,

$$
\begin{aligned}
\left(\mathcal{T}_{i}\left(u_{1}, u_{2}\right)\right)\left(t_{1}, t_{2}\right) & \\
:= & \frac{t_{i}^{\alpha_{i}-1}}{\left[\left(T+\alpha_{i}\right) \frac{\alpha_{i}-1}{\chi_{i}}-\chi_{i} \eta_{i}^{\alpha_{i}-1}\right] \Gamma\left(\alpha_{i}\right)} \\
& \times\left\{\sum_{s=0}^{T}\left(T+\alpha_{i}-\sigma(s)\right) \frac{\alpha_{i}-1}{{ }^{2}} F_{i}\left[s+\alpha_{i}-1, t_{j}, u_{i}\left(s+\alpha_{i}-1\right), u_{j}\left(t_{j}\right)\right]\right. \\
& \left.-\chi_{i} \sum_{s=0}^{\eta_{i}-\alpha_{i}}\left(\eta_{i}-\sigma(s)\right)^{\frac{\alpha_{i}-1}{}} F_{i}\left[s+\alpha_{i}-1, t_{j}, u_{i}\left(s+\alpha_{i}-1\right), u_{j}\left(t_{j}\right)\right]\right\} \\
& -\frac{1}{\Gamma\left(\alpha_{i}\right)} \sum_{s=0}^{t_{i}-\alpha_{1}}\left(t_{i}-\sigma(s)\right) \frac{\alpha_{i}-1}{} F_{i}\left[s+\alpha_{i}-1, t_{j}, u_{i}\left(s+\alpha_{i}-1\right), u_{j}\left(t_{j}\right)\right]
\end{aligned}
$$

for $t_{i} \in \mathbb{N}_{\alpha_{i}-3, T+\alpha_{i}}$ and $t_{j} \in \mathbb{N}_{\alpha_{j}-1, T+\alpha_{j}-1}$. Obviously, problem (1.1)-(1.2) has a solution if and only if the operator $\mathcal{T}$ has a fixed point.

Theorem 3.1 Suppose that $(A 1)-(A 4)$ hold. In addition, suppose there exist constants $M_{i}, N_{i}>0$ for $i=1,2$ such that

$$
\left|F_{i}\left[t_{1}, t_{2}, u_{1}, u_{2}\right]-F_{i}\left[t_{1}, t_{2}, v_{1}, v_{2}\right]\right| \leq M_{i}\left|u_{1}-v_{1}\right|+N_{i}\left|u_{2}-v_{2}\right|
$$


for all $t_{i} \in \mathbb{N}_{\alpha_{i}-1, T+\alpha_{i}-1}$ and $\left(u_{1}, u_{2}\right),\left(v_{1}, v_{2}\right) \in \mathcal{U}$. If

$$
L_{1} \Omega_{1}+L_{2} \Omega_{2}<1
$$

where

$$
\begin{aligned}
& L_{i}=\max \left\{M_{i}, N_{i}\right\} \\
& \Omega_{i}=\frac{\lambda_{i}}{\Gamma\left(\alpha_{i}+1\right)}\left[\left|\frac{\left(T+\alpha_{i}\right)^{\frac{\alpha_{i}}{2}}-\chi_{i} \eta_{i}^{\frac{\alpha_{i}}{i}}}{\left(T+\alpha_{i}\right)^{\frac{\alpha_{i}-1}{2}}-\chi_{i} \eta_{i}^{\frac{\alpha_{i}-1}{i}}}\right|\left(T+\alpha_{i}\right) \frac{\alpha_{i}-1}{}+\left(T+\alpha_{i}\right)^{\frac{\alpha_{i}}{}}\right],
\end{aligned}
$$

then problem (1.1)-(1.2) has a unique solution.

Proof Let us prove that $\mathcal{T}$ is a contraction mapping. For $i, j \in\{1,2\}, i \neq j$, denote

$$
\begin{aligned}
\mathcal{F}_{i}|u-v|\left(s, t_{j}\right):= & \mid F_{i}\left[s+\alpha_{i}-1, t_{j}, u_{i}\left(s+\alpha_{i}-1\right), u_{j}\left(t_{j}\right)\right] \\
& -F_{i}\left[s+\alpha_{i}-1, t_{j}, v_{i}\left(s+\alpha_{i}-1\right), v_{j}\left(t_{j}\right)\right] \mid
\end{aligned}
$$

For $t_{i} \in \mathbb{N}_{\alpha_{i}-3, T+\alpha_{i}}, t_{j} \in \mathbb{N}_{\alpha_{j}-1, T+\alpha_{j}-1}$, and $\left(u_{1}, u_{2}\right),\left(v_{1}, v_{2}\right) \in \mathcal{U}$, we find that

$$
\begin{aligned}
& \left|\left(\mathcal{T}_{i}\left(u_{1}, u_{2}\right)\right)\left(t_{1}, t_{2}\right)-\left(\mathcal{T}_{i}\left(v_{1}, v_{2}\right)\right)\left(t_{1}, t_{2}\right)\right| \\
& \leq \frac{\lambda_{i} t_{i}^{\alpha_{i}-1}}{\left[\left(T+\alpha_{i}\right) \frac{\alpha_{i}-1}{-}-\chi_{i} \eta_{i}^{\frac{\alpha_{i}-1}{2}}\right] \Gamma\left(\alpha_{i}\right)}\left|\sum_{s=0}^{T}\left(T+\alpha_{i}-\sigma(s)\right) \frac{\alpha_{i}-1}{} \mathcal{F}_{i}\right| u-v \mid\left(s, t_{j}\right) \\
& -\chi_{i} \sum_{s=0}^{\eta_{i}-\alpha_{i}}\left(\eta_{i}-\sigma(s)\right) \frac{\alpha_{i}-1}{} \mathcal{F}_{i}|u-v|\left(s, t_{j}\right)\left|+\frac{\lambda_{i}}{\Gamma\left(\alpha_{i}\right)} \sum_{s=0}^{t_{i}-\alpha_{1}}\left(t_{i}-\sigma(s)\right) \frac{\alpha_{i}-1}{\mathcal{F}_{i}}\right| u-v \mid\left(s, t_{j}\right) \\
& \leq \frac{\lambda_{i}\left[M_{i}\left|u_{1}+v_{1}\right|+N_{i}\left|u_{2}-v_{2}\right|\right]}{\Gamma\left(\alpha_{i}\right)}\left[\frac{\left(T+\alpha_{i}\right) \frac{\alpha_{i}-1}{\alpha_{i}^{\alpha_{i}-1}}-\chi_{i} \eta_{i}^{\alpha_{i}-1}}{t_{i}}\right. \\
& \left.\times\left|\sum_{s=0}^{T}\left(T+\alpha_{i}-\sigma(s)\right) \frac{\alpha_{i}-1}{2}-\chi_{i} \sum_{s=0}^{\eta_{i}-\alpha_{i}}\left(\eta_{i}-\sigma(s)\right) \frac{\alpha_{i}-1}{}\right|+\sum_{s=0}^{t_{i}-\alpha_{1}}\left(t_{i}-\sigma(s)\right) \frac{\alpha_{i}-1}{}\right] \\
& \leq \frac{\lambda_{i} L_{i}\left[\left|u_{1}+v_{1}\right|+\left|u_{2}-v_{2}\right|\right]}{\Gamma\left(\alpha_{i}+1\right)}\left[\left|\frac{\left(T+\alpha_{i}\right) \frac{\alpha_{i}}{-}-\chi_{i} \eta_{i}^{\frac{\alpha_{i}}{2}}}{\left(T+\alpha_{i}\right) \frac{\alpha_{i}-1}{-}-\chi_{i} \eta_{i}^{\frac{\alpha_{i}-1}{}}}\right|\left(T+\alpha_{i}\right)^{\alpha_{i}-1}+\left(T+\alpha_{i}\right) \frac{\alpha_{i}}{}\right] \\
& \leq L_{i} \Omega_{i}\left\|\left(u_{1}, v-1, u_{2}-v_{2}\right)\right\|_{\mathcal{U}}
\end{aligned}
$$

Therefore

$$
\begin{aligned}
\left\|\left(\mathcal{T}\left(u_{1}, u_{2}\right)\right)-\left(\mathcal{T}\left(v_{1}, v_{2}\right)\right)\right\|_{\mathcal{U}} & =\left\|\left(\mathcal{T}_{1}\left(u_{1}, u_{2}\right)-\mathcal{T}_{1}\left(v_{1}, v_{2}\right), \mathcal{T}_{2}\left(u_{1}, u_{2}\right)-\mathcal{T}_{2}\left(v_{1}, v_{2}\right)\right)\right\|_{\mathcal{U}} \\
& =\left\|\mathcal{T}_{1}\left(u_{1}, u_{2}\right)-\mathcal{T}_{1}\left(v_{1}, v_{2}\right)\right\|+\left\|\mathcal{T}_{2}\left(u_{1}, u_{2}\right)-\mathcal{T}_{2}\left(v_{1}, v_{2}\right)\right\| \\
& \leq\left(L_{1} \Omega_{1}+L_{2} \Omega_{2}\right)\left\|\left(u_{1}, v-1, u_{2}-v_{2}\right)\right\|_{\mathcal{U}} .
\end{aligned}
$$

By (3.4) we find that $\mathcal{T}$ is a contraction. Therefore by the Banach fixed point theorem $\mathcal{T}$ has a fixed point, which is a unique solution of problem (1.1)-(1.2). 


\section{Existence and multiplicity of positive solutions}

In this section, we apply the Krasnoselskii fixed point theorem for a cone map to prove the existence and multiplicity of positive solutions for problem (1.1)-(1.2). The product space $\mathcal{U}$ and the norm $\left\|\left(u_{1}, u_{2}\right)\right\|_{\mathcal{U}}$ are defined in Sect. 3. Moreover, we define the cone $\mathcal{P} \subset \mathcal{U}$ as

$$
\mathcal{P}:=\left\{\left(u_{1}, u_{2}\right) \in \mathcal{U}: u_{1}, u_{2} \geq 0 \text { and } \min _{t_{i} \in \mathbb{N}_{\alpha_{i}-3, T+\alpha_{i}}}\left[u_{1}\left(t_{1}\right)+u_{2}\left(t_{2}\right)\right] \geq \theta\left\|\left(u_{1}, u_{2}\right)\right\|_{\mathcal{U}}\right\},
$$

where $\theta:=\min \left\{\theta_{1}, \theta_{2}\right\}$ with $\theta_{1}, \theta_{2}$ defined in (2.14).

Next, by Corollary 2.1 we also define the operator $\mathcal{T}: \mathcal{U} \rightarrow \mathcal{U}$ by

$$
\left(\mathcal{T}\left(u_{1}, u_{2}\right)\right)\left(t_{1}, t_{2}\right)=\left(\left(\mathcal{T}_{1}\left(u_{1}, u_{2}\right)\right)\left(t_{1}, t_{2}\right),\left(\mathcal{T}_{2}\left(u_{1}, u_{2}\right)\right)\left(t_{1}, t_{2}\right)\right)
$$

and for $i, j \in\{1,2\}, i \neq j$,

$$
\left(\mathcal{T}_{i}\left(u_{1}, u_{2}\right)\right)\left(t_{1}, t_{2}\right):=\lambda_{i} \sum_{s=0}^{T} G_{i}\left(t_{i}, s\right) F_{i}\left[s+\alpha_{i}-1, t_{j}, u_{i}\left(s+\alpha_{i}-1\right), u_{j}\left(t_{j}\right)\right]
$$

for $t_{i} \in \mathbb{N}_{\alpha_{i}-3, T+\alpha_{i}}$ and $t_{j} \in \mathbb{N}_{\alpha_{j}-1, T+\alpha_{j-1}}$. The positive solutions of problem (1.1)-(1.2) and the fixed points of the operator $\mathcal{T}$ in the cone $\mathcal{P}$ coincide.

Lemma 4.1 If $(A 1)-(A 4)$ hold, then $\mathcal{T}(\mathcal{P}) \subset \mathcal{P}$, and $\mathcal{T}: \mathcal{P} \rightarrow \mathcal{P}$ is a completely continuous operator.

Proof The continuity of $\mathcal{T}$ is obvious. To prove $\mathcal{T}(\mathcal{P}) \subset \mathcal{P}$, we choose $\left(u_{1}, u_{2}\right) \in \mathcal{P}$. Since, for $i=1,2, G_{i}\left(t_{i}, s\right) \leq G_{i}\left(s+\alpha_{i}-1, s\right)$ for $s \in \mathbb{N}_{0, T}$ and $G_{i}\left(t_{i}, s\right) \geq \theta_{i} G_{i}(s+\alpha-1, s)$ for $t_{i} \in$ $\left[\frac{1}{4}\left(T+\alpha_{i}\right), \frac{3}{4}\left(T+\alpha_{i}\right)\right]$, we have

$$
\begin{aligned}
& \min _{t_{i} \in\left[\frac{1}{4}\left(T+\alpha_{i}\right), \frac{3}{4}\left(T+\alpha_{i}\right)\right]} \mathcal{T}_{1}\left(u_{1}, u_{2}\right)\left(t_{1}, t_{2}\right) \\
& \geq \lambda_{1} \theta_{1} \sum_{s=0}^{T} G_{i}\left(s+\alpha_{i}-1, s\right) F_{1}\left[s+\alpha_{i}-1, t_{2}, u_{1}\left(s+\alpha_{i}-1\right), u_{2}\left(t_{2}\right)\right] \\
& \geq \theta_{1}\left\|\mathcal{T}_{1}\left(u_{1}, u_{2}\right)\right\|
\end{aligned}
$$

Similarly, $\min _{t_{i} \in\left[\frac{1}{4}\left(T+\alpha_{i}\right), \frac{3}{4}\left(T+\alpha_{i}\right)\right]} \mathcal{T}_{2}\left(u_{1}, u_{2}\right)\left(t_{1}, t_{2}\right) \geq \theta_{2}\left\|\mathcal{T}_{2}\left(u_{1}, u_{2}\right)\right\|$.

Thus

$$
\begin{aligned}
& \min _{t_{i} \in\left[\frac{1}{4}\left(T+\alpha_{i}\right), \frac{3}{4}\left(T+\alpha_{i}\right)\right]}\left(\mathcal{T}_{1}\left(u_{1}, u_{2}\right)\left(t_{1}, t_{2}\right)+\mathcal{T}_{2}\left(u_{1}, u_{2}\right)\left(t_{1}, t_{2}\right)\right) \\
& \quad \geq \min _{t_{i} \in\left[\frac{1}{4}\left(T+\alpha_{i}\right), \frac{3}{4}\left(T+\alpha_{i}\right)\right]} \mathcal{T}_{1}\left(u_{1}, u_{2}\right)\left(t_{1}, t_{2}\right)+\min _{t_{i} \in\left[\frac{1}{4}\left(T+\alpha_{i}\right), \frac{3}{4}\left(T+\alpha_{i}\right)\right]} \mathcal{T}_{2}\left(u_{1}, u_{2}\right)\left(t_{1}, t_{2}\right) \\
& \geq \theta\left\|\left(\mathcal{T}_{1}\left(u_{1}, u_{2}\right), \mathcal{T}_{2}\left(u_{1}, u_{2}\right)\right)\right\|_{\mathcal{U}} .
\end{aligned}
$$

Since $G_{i}\left(t_{i}, s\right) \geq 0$ for all $\left(t_{i}, s\right) \in \mathbb{N}_{\alpha_{i}-3, T+\alpha_{i}} \times \mathbb{N}_{0, T}$ and (A1)-(A4) hold, we conclude that $\mathcal{T}(\mathcal{P}) \subset \mathcal{P}$. It is easy to show that $\mathcal{T}$ is uniformly bounded. By the Arzelá-Ascoli theorem we can conclude that $\mathcal{T}: \mathcal{P} \rightarrow \mathcal{P}$ is a completely continuous operator. 
Theorem 4.1 Suppose assumptions $(A 1)-(A 4)$ hold. Then for $\lambda_{1}, \lambda_{2}>0$, problem (1.1)(1.2) has at least one positive solution in the following cases:

(a) $F_{1}^{0}=F_{2}^{0}=0$, and either $F_{1}^{\infty}=\infty$ or $F_{2}^{\infty}=\infty$ (superlinear);

(b) $F_{1}^{\infty}=F_{2}^{\infty}=0$, and either $F_{1}^{0}=\infty$ or $F_{2}^{0}=\infty$ (sublinear).

Proof (a) Since, $F_{i}^{0}=0, i=1,2$, we choose $K_{1}>0$ such that $F_{i}\left[t_{1}, t_{2}, u_{1}, u_{2}\right] \leq \varepsilon\left(u_{1}+u_{2}\right)$ for $0<u_{1}+u_{2} \leq K_{1}$ and $t_{i} \in \mathbb{N}_{\alpha_{i}-1, T+\alpha_{i}-1}$, where the constant $\varepsilon>0$ satisfies

$$
2 \varepsilon \lambda_{i} \sum_{s=0}^{T} G_{i}\left(s+\alpha_{i}-1, s\right) \leq 1 .
$$

Set $\Omega_{1}=\left\{\left(u_{1}, u_{2}\right) \in \mathcal{U}:\left\|\left(u_{1}, u_{2}\right)\right\|_{\mathcal{U}}<K_{1}\right\}$. If $\left(u_{1}, u_{2}\right) \in \mathcal{P} \cap \partial \Omega_{1}$ and $\left\|\left(u_{1}, u_{2}\right)\right\|_{\mathcal{U}}=K_{1}$, then we have

$$
\begin{aligned}
\mathcal{T}_{1}\left(u_{1}, u_{2}\right)\left(t_{1}, t_{2}\right) & \leq \lambda_{1} \sum_{s=0}^{T} G_{1}\left(t_{1}, s\right) F_{1}\left[s-\alpha_{1}-1, t_{2}, u_{1}\left(s-\alpha_{1}-1\right), u_{2}\left(t_{2}\right)\right] \\
& \leq \varepsilon \lambda_{1} \sum_{s=0}^{T} G_{1}\left(s+\alpha_{i}-1, s\right)\left(u_{1}+u_{2}\right) \\
& \leq \varepsilon \lambda_{1}\left(\left\|u_{1}\right\|+\left\|u_{2}\right\|\right) \sum_{s=0}^{T} G_{1}\left(s+\alpha_{i}-1, s\right) \\
& \leq \frac{1}{2}\left\|\left(u_{1}, u_{2}\right)\right\|_{\mathcal{U}} .
\end{aligned}
$$

Similarly, $\mathcal{T}_{2}\left(u_{1}, u_{2}\right)\left(t_{1}, t_{2}\right) \leq \frac{1}{2}\left\|\left(u_{1}, u_{2}\right)\right\|_{\mathcal{U}}$. Therefore

$$
\left\|\mathcal{T}\left(u_{1}, u_{2}\right)\right\|_{\mathcal{U}}=\left\|\left(\mathcal{T}_{1}\left(u_{1}, u_{2}\right), \mathcal{T}_{2}\left(u_{1}, u_{2}\right)\right)\right\|_{\mathcal{U}}=\left\|\mathcal{T}_{1}\left(u_{1}, u_{2}\right)\right\|+\left\|\mathcal{T}_{2}\left(u_{1}, u_{2}\right)\right\| \leq\left\|\left(u_{1}, u_{2}\right)\right\|_{\mathcal{U}}
$$

for $\left(u_{1}, u_{2}\right) \in \mathcal{P} \cap \partial \Omega_{1}$.

If $F_{1}^{\infty}=\infty$, then there exists $\hat{K}>0$ such that $F_{1}\left[t_{1}, t_{2}, u_{1}, u_{2}\right] \geq \hat{\varepsilon}\left(u_{1}+u_{2}\right)$ for $u_{1}+u_{2} \geq \hat{K}_{1}$ and $t_{i} \in \mathbb{N}_{\alpha_{i}-1, T+\alpha_{i}-1}$, where the constant $\hat{\varepsilon}>0$ satisfies

$$
\hat{\varepsilon} \lambda_{1} \sum_{s=0}^{T} G_{1}\left(s+\alpha_{1}-1, s\right) \geq 1 .
$$

Let $K_{2}=\max \left\{2 K_{1}, \frac{\hat{K}}{\theta_{1}}\right\}$ and set $\Omega_{2}=\left\{\left(u_{1}, u_{2}\right) \in \mathcal{U}:\left\|\left(u_{1}, u_{2}\right)\right\|_{\mathcal{U}}<K_{2}\right\}$. For $\left(u_{1}, u_{2}\right) \in \mathcal{P} \cap$ $\partial \Omega_{2}$, we have $\min _{t_{i} \in\left[\frac{1}{4}\left(T+\alpha_{i}\right), \frac{3}{4}\left(T+\alpha_{i}\right)\right]}\left(\left(u_{1}\left(t_{1}\right)+u_{2}\left(t_{2}\right)\right) \geq \theta_{i}\left\|\left(u_{1}, u_{2}\right)\right\|_{\mathcal{U}} \geq \hat{K}\right.$. For all $t_{i} \in\left[\frac{1}{4}(T+\right.$ $\left.\left.\alpha_{i}\right), \frac{3}{4}\left(T+\alpha_{i}\right)\right]$, we get

$$
\begin{aligned}
& \min _{t_{i} \in\left[\frac{1}{4}\left(T+\alpha_{i}\right), \frac{3}{4}\left(T+\alpha_{i}\right)\right]} \mathcal{T}_{1}\left(u_{1}, u_{2}\right)\left(t_{1}, t_{2}\right) \\
& \geq \min _{t_{i} \in\left[\frac{1}{4}\left(T+\alpha_{i}\right), \frac{3}{4}\left(T+\alpha_{i}\right)\right]} \lambda_{1} \sum_{s=0}^{T} G_{1}\left(t_{1}, s\right) F_{1}\left[s-\alpha_{1}-1, t_{2}, u_{1}\left(s-\alpha_{1}-1\right), u_{2}\left(t_{2}\right)\right]
\end{aligned}
$$




$$
\begin{aligned}
& \geq \hat{\varepsilon} \lambda_{1} \sum_{s=0}^{T} G_{1}\left(s+\alpha_{i}-1, s\right)\left(u_{1}+u_{2}\right) \\
& \geq \hat{\varepsilon} \lambda_{1} \theta_{1}\left\|\left(u_{1}, u_{2}\right)\right\|_{\mathcal{U}} \sum_{s=0}^{T} G_{1}\left(s+\alpha_{i}-1, s\right) \\
& \geq\left\|\left(u_{1}, u_{2}\right)\right\|_{\mathcal{U}} .
\end{aligned}
$$

Therefore

$$
\left\|\mathcal{T}\left(u_{1}, u_{2}\right)\right\|_{\mathcal{U}}=\left\|\left(\mathcal{T}_{1}\left(u_{1}, u_{2}\right), \mathcal{T}_{2}\left(u_{1}, u_{2}\right)\right)\right\|_{\mathcal{U}}=\left\|\mathcal{T}_{1}\left(u_{1}, u_{2}\right)\right\|+\left\|\mathcal{T}_{2}\left(u_{1}, u_{2}\right)\right\| \geq\left\|\left(u_{1}, u_{2}\right)\right\|_{\mathcal{U}}
$$

for $\left(u_{1}, u_{2}\right) \in \mathcal{P} \cap \partial \Omega_{2}$. An analogous estimate holds for $F_{2}^{\infty}=\infty$.

By Theorem 1.1(i), $\mathcal{T}$ has a fixed point $\left(u_{1}, u_{2}\right) \in \mathcal{P} \cap \partial\left(\bar{\Omega}_{2} \backslash \Omega_{1}\right)$ such that $K_{1} \leq$ $\left\|\left(u_{1}, u_{2}\right)\right\|_{\mathcal{U}} \leq K_{2}$ and problem (1.1)-(1.2) has a positive solution.

(b) If $F_{1}^{0}=\infty$, then we choose $K_{1}>0$ such that $F_{1}\left[t_{1}, t_{2}, u_{1}, u_{2}\right] \geq \tilde{\varepsilon}\left(u_{1}+u_{2}\right)$ for $0<u_{1}+$ $u_{2} \leq K_{2}$ and $t_{i} \in \mathbb{N}_{\alpha_{i}-1, T+\alpha_{i}-1}$, where the constant $\tilde{\varepsilon}>0$ satisfies

$$
\tilde{\varepsilon} \lambda_{1} \theta_{1} \sum_{s=0}^{T} G_{1}\left(s+\alpha_{1}-1, s\right) \geq 1 .
$$

If $\left(u_{1}, u_{2}\right) \in \mathcal{P} \cap \partial \Omega_{1}$ and $\left\|\left(u_{1}, u_{2}\right)\right\|_{\mathcal{U}}=K_{1}$, then for all $t_{i} \in\left[\frac{1}{4}\left(T+\alpha_{i}\right), \frac{3}{4}\left(T+\alpha_{i}\right)\right]$, we have

$$
\begin{aligned}
& \min _{t_{i} \in\left[\frac{1}{4}\left(T+\alpha_{i}\right), \frac{3}{4}\left(T+\alpha_{i}\right)\right]} \mathcal{T}_{1}\left(u_{1}, u_{2}\right)\left(t_{1}, t_{2}\right) \\
& \geq \min _{t_{i} \in\left[\frac{1}{4}\left(T+\alpha_{i}\right), \frac{3}{4}\left(T+\alpha_{i}\right)\right]} \lambda_{1} \sum_{s=0}^{T} G_{1}\left(t_{1}, s\right) F_{1}\left[s-\alpha_{1}-1, t_{2}, u_{1}\left(s-\alpha_{1}-1\right), u_{2}\left(t_{2}\right)\right] \\
& \geq \tilde{\varepsilon} \lambda_{1} \sum_{s=0}^{T} G_{1}\left(s+\alpha_{i}-1, s\right)\left(u_{1}+u_{2}\right) \\
& \geq \tilde{\varepsilon} \lambda_{1} \theta_{1}\left\|\left(u_{1}, u_{2}\right)\right\|_{\mathcal{U}} \sum_{s=0}^{T} G_{1}\left(s+\alpha_{i}-1, s\right) \\
& \geq\left\|\left(u_{1}, u_{2}\right)\right\|_{\mathcal{U}} .
\end{aligned}
$$

Therefore

$$
\left\|\mathcal{T}\left(u_{1}, u_{2}\right)\right\|_{\mathcal{U}}=\left\|\left(\mathcal{T}_{1}\left(u_{1}, u_{2}\right), \mathcal{T}_{2}\left(u_{1}, u_{2}\right)\right)\right\|_{\mathcal{U}}=\left\|\mathcal{T}_{1}\left(u_{1}, u_{2}\right)\right\|+\left\|\mathcal{T}_{2}\left(u_{1}, u_{2}\right)\right\| \geq\left\|\left(u_{1}, u_{2}\right)\right\|_{\mathcal{U}}
$$

for $\left(u_{1}, u_{2}\right) \in \mathcal{P} \cap \partial \Omega_{2}$. An analogous estimate holds for $F_{2}^{0}=\infty$.

For $i=1,2$, set $F_{i}^{*}\left(t_{i}\right)=\max _{0 \leq u_{1}+u_{2} \leq t_{i}} F_{i}\left[t_{1}, t_{2}, u_{1}, u_{2}\right]$. Then $F_{i}^{*}$ are nondecreasing in their respective arguments. In addition, from $F_{i}^{\infty}=0$ we see that $\lim _{t_{i} \rightarrow \infty} \frac{F_{i}^{*}\left(t_{i}\right)}{t_{i}}=0$. Hence there exist $K_{2}>2 K_{1}$ such that $F_{i}^{*}\left(t_{i}\right) \leq \varepsilon t_{i}$, where the constant $\varepsilon>0$ satisfies

$$
2 \varepsilon \lambda_{i} \sum_{s=0}^{T} G_{i}\left(s+\alpha_{i}-1, s\right) \leq 1 .
$$


If $\left(u_{1}, u_{2}\right) \in \mathcal{P} \cap \partial \Omega_{2}$ and $\left\|\left(u_{1}, u_{2}\right)\right\|_{\mathcal{U}}=K_{2}$, then we have

$$
\begin{aligned}
\mathcal{T}_{1}\left(u_{1}, u_{2}\right)\left(t_{1}, t_{2}\right) & \leq \lambda_{1} \sum_{s=0}^{T} G_{1}\left(t_{1}, s\right) F_{1}\left[s-\alpha_{1}-1, t_{2}, u_{1}\left(s-\alpha_{1}-1\right), u_{2}\left(t_{2}\right)\right] \\
& \leq \lambda_{1} \sum_{s=0}^{T} G_{1}\left(s+\alpha_{i}-1, s\right) F_{1}^{*}\left(K_{2}\right) \\
& \leq \varepsilon \lambda_{1} K_{2} \sum_{s=0}^{T} G_{1}\left(s+\alpha_{i}-1, s\right) \\
& \leq \frac{1}{2}\left\|\left(u_{1}, u_{2}\right)\right\|_{\mathcal{U}} .
\end{aligned}
$$

Similarly, $\mathcal{T}_{2}\left(u_{1}, u_{2}\right)\left(t_{1}, t_{2}\right) \leq \frac{1}{2}\left\|\left(u_{1}, u_{2}\right)\right\| \mathcal{u}$. Therefore

$$
\left\|\mathcal{T}\left(u_{1}, u_{2}\right)\right\|_{\mathcal{U}}=\left\|\left(\mathcal{T}_{1}\left(u_{1}, u_{2}\right), \mathcal{T}_{2}\left(u_{1}, u_{2}\right)\right)\right\|_{\mathcal{U}}=\left\|\mathcal{T}_{1}\left(u_{1}, u_{2}\right)\right\|+\left\|\mathcal{T}_{2}\left(u_{1}, u_{2}\right)\right\| \leq\left\|\left(u_{1}, u_{2}\right)\right\|_{\mathcal{U}}
$$

for $\left(u_{1}, u_{2}\right) \in \mathcal{P} \cap \partial \Omega_{2}$.

By Theorem 1.1(ii), $\mathcal{T}$ has a fixed point, and thus problem (1.1)-(1.2) has a positive solution $\left(u_{1}, u_{2}\right) \in \mathcal{P} \cap \partial\left(\bar{\Omega}_{2} \backslash \Omega_{1}\right)$.

Theorem 4.2 Suppose assumptions (A1)-(A4) hold.

(a) If $F_{1}^{0}=F_{2}^{0}=F_{1}^{\infty}=F_{2}^{\infty}=0$, then there exists $\delta_{1}>0$ such that problem (1.1)-(1.2) has at least two positive solutions for all $\lambda_{1}, \lambda_{2} \geq \delta_{1}$.

(b) If either $F_{1}^{0}=\infty$ or $F_{2}^{0}=\infty$ and either $F_{1}^{\infty}=0$ or $F_{2}^{\infty}=0$, then there exists $\delta_{2}>0$ such that problem (1.1)-(1.2) has at least two positive solutions for all $\lambda_{1}, \lambda_{2} \leq \delta_{2}$.

Proof $(a)$ For $\left(u_{1}, u_{2}\right) \in \mathcal{P}$ such that $\left\|\left(u_{1}, u_{2}\right)\right\|_{\mathcal{U}}=\ell$, let

$$
\begin{aligned}
m(\ell)= & \min \left\{\lambda_{1} \sum_{s=0}^{T} G_{1}\left(t_{1}, s\right) F_{1}\left[s-\alpha_{1}-1, t_{2}, u_{1}\left(s-\alpha_{1}-1\right), u_{2}\left(t_{2}\right)\right],\right. \\
& \left.\lambda_{2} \sum_{s=0}^{T} G_{2}\left(t_{1}, s\right) F_{2}\left[t_{1}, s-\alpha_{2}-1, u_{1}\left(t_{1}\right), u_{2}\left(s-\alpha_{2}-1\right)\right]\right\} .
\end{aligned}
$$

By assumption $m(\ell)>0$ for $\ell>0$. Choose two numbers $0<K_{3}<K_{4}$, and let

$$
\begin{aligned}
& \delta_{1}=\max \left\{\frac{K_{3}}{2 m\left(K_{3}\right)}, \frac{K_{4}}{2 m\left(K_{4}\right)}\right\}, \\
& \Omega_{i}=\left\{\left(u_{1}, u_{2}\right) \in \mathcal{U}:\left\|\left(u_{1}, u_{2}\right)\right\|_{\mathcal{U}}<K_{i}\right\} \quad(i=1,2,3,4) .
\end{aligned}
$$

Then, for $\lambda_{1}, \lambda_{2} \geq \delta_{1}$ and $\left(u_{1}, u_{2}\right) \in \mathcal{P} \cap \partial \Omega_{i}(i=3,4)$ such that $\left\|\left(u_{1}, u_{2}\right)\right\|_{\mathcal{U}}=K_{i}$, we have

$$
\begin{aligned}
& \min _{t_{i} \in\left[\frac{1}{4}\left(T+\alpha_{i}\right), \frac{3}{4}\left(T+\alpha_{i}\right)\right]} \mathcal{T}_{1}\left(u_{1}, u_{2}\right)\left(t_{1}, t_{2}\right) \\
& \geq \tilde{\varepsilon} \lambda_{1} \sum_{s=0}^{T} G_{1}\left(t_{1}, s\right) F_{1}\left[s-\alpha_{1}-1, t_{2}, u_{1}\left(s-\alpha_{1}-1\right), u_{2}\left(t_{2}\right)\right]
\end{aligned}
$$




$$
\begin{aligned}
& \geq \lambda_{1} m\left(K_{i}\right) \\
& \geq \frac{K_{i}}{2} \quad(i=3,4) .
\end{aligned}
$$

Similarly, $\min _{t_{i} \in\left[\frac{1}{4}\left(T+\alpha_{i}\right), \frac{3}{4}\left(T+\alpha_{i}\right)\right]} \mathcal{T}_{2}\left(u_{1}, u_{2}\right)\left(t_{1}, t_{2}\right) \geq \frac{K_{i}}{2}(i=3,4)$.

This implies that

$$
\left\|\mathcal{T}\left(u_{1}, u_{2}\right)\right\|_{\mathcal{U}}=\left\|\mathcal{T}_{1}\left(u_{1}, u_{2}\right)\right\|+\left\|\mathcal{T}_{2}\left(u_{1}, u_{2}\right)\right\| \geq K_{i}=\left\|\left(u_{1}, u_{2}\right)\right\|_{\mathcal{U}}
$$

for $\left(u_{1}, u_{2}\right) \in \mathcal{P} \cap \partial \Omega_{i}(i=3,4)$.

Since $F_{1}^{0}=F_{2}^{0}=F_{1}^{\infty}=F_{2}^{\infty}=0$, it follows from the proof of Theorem 4.1(a,b) that we can choose $K_{1}<\frac{K_{3}}{2}$ and $K_{2}>2 K_{4}$ such that

$$
\left\|\mathcal{T}\left(u_{1}, u_{2}\right)\right\|_{\mathcal{U}} \leq\left\|\left(u_{1}, u_{2}\right)\right\|_{\mathcal{U}}
$$

for $\left(u_{1}, u_{2}\right) \in \mathcal{P} \cap \partial \Omega_{i}(i=1,2)$.

Applying Theorem 1.1 to $\Omega_{1}, \Omega_{3}$ and $\Omega_{3}, \Omega_{4}$, we have a positive solution $\left(u_{1}, u_{2}\right)$ such that $K_{1} \leq\left\|\left(u_{1}, u_{2}\right)\right\|_{\mathcal{U}} \leq K_{3}$, and another positive solution $\left(v_{1}, v_{2}\right)$ such that $K_{4} \leq$ $\left\|\left(v_{1}, v_{2}\right)\right\|_{\mathcal{U}} \leq K_{2}$. Since $K_{3}<K_{4}$, these two solutions are distinct.

(b) For $\left(u_{1}, u_{2}\right) \in \mathcal{P}$ and $\left\|\left(u_{1}, u_{2}\right)\right\|_{\mathcal{U}}=L$, let

$$
\begin{aligned}
M(L)= & \max \left\{\lambda_{1} \sum_{s=0}^{T} G_{1}\left(t_{1}, s\right) F_{1}\left[s-\alpha_{1}-1, t_{2}, u_{1}\left(s-\alpha_{1}-1\right), u_{2}\left(t_{2}\right)\right],\right. \\
& \left.\lambda_{2} \sum_{s=0}^{T} G_{2}\left(t_{1}, s\right) F_{2}\left[t_{1}, s-\alpha_{2}-1, u_{1}\left(t_{1}\right), u_{2}\left(s-\alpha_{2}-1\right)\right]\right\} .
\end{aligned}
$$

By assumption $M(L)>0$ for $L>0$. Choose two numbers $0<K_{3}<K_{4}$, and let

$$
\delta_{2}=\max \left\{\frac{K_{3}}{2 M\left(K_{3}\right)}, \frac{K_{4}}{2 M\left(K_{4}\right)}\right\} .
$$

Then, for $\lambda_{1}, \lambda_{2} \leq \delta_{2}$ and $\left(u_{1}, u_{2}\right) \in \mathcal{P} \cap \partial \Omega_{i}(i=3,4)$ such that $\left\|\left(u_{1}, u_{2}\right)\right\|_{\mathcal{U}}=K_{i}$, we have

$$
\mathcal{T}_{1}\left(u_{1}, u_{2}\right)\left(t_{1}, t_{2} \leq \lambda_{1} M\left(H_{i}\right) \leq \frac{K_{i}}{2} \quad \text { and } \quad \mathcal{T}_{2}\left(u_{1}, u_{2}\right)\left(t_{1}, t_{2}\right) \leq \lambda_{2} M\left(H_{i}\right) \leq \frac{K_{i}}{2} \quad(i=3,4) .\right.
$$

This implies that

$$
\left\|\mathcal{T}\left(u_{1}, u_{2}\right)\right\|_{\mathcal{U}} \leq K_{i}=\left\|\left(u_{1}, u_{2}\right)\right\|_{\mathcal{U}}
$$

for $\left(u_{1}, u_{2}\right) \in \mathcal{P} \cap \partial \Omega_{i}(i=3,4)$.

Since either $F_{1}^{0}=\infty$ or $F_{2}^{0}=\infty$ and either $F_{1}^{\infty}=0$ or $F_{2}^{\infty}=0$, it follows from the proof of Theorem 4.1(a,b) that we can choose $K_{1}<\frac{K_{3}}{2}$ and $K_{2}>2 K_{4}$ such that

$$
\left\|\mathcal{T}\left(u_{1}, u_{2}\right)\right\|_{\mathcal{U}} \geq\left\|\left(u_{1}, u_{2}\right)\right\|_{\mathcal{U}}
$$

for $\left(u_{1}, u_{2}\right) \in \mathcal{P} \cap \partial \Omega_{i}(i=1,2)$.

Once again, we obtain the existence of two distinct positive solutions. 
By the same arguments as in Theorem 4.2 we obtain the following theorem.

Theorem 4.3 Suppose assumptions (A1)-(A4) hold.

(a) If $F_{1}^{0}=F_{2}^{0}=0$ or $F_{1}^{\infty}=F_{2}^{\infty}=0$, then there exists $\delta_{3}>0$ such that problem (1.1)-(1.2) has at least two positive solutions for all $\lambda_{1}, \lambda_{2} \geq \delta_{3}$.

(b) If $F_{1}^{0}=\infty$ or $F_{2}^{0}=\infty$, or if $F_{1}^{\infty}=\infty$ or $F_{2}^{\infty}=\infty$, then there exists $\delta_{4}>0$ such that problem (1.1)-(1.2) has at least two positive solutions for all $\lambda_{1}, \lambda_{2} \leq \delta_{2}$.

\section{Examples}

In this section, we provide some examples to illustrate our main results.

Example 1 Consider the following system of fractional difference equations with parameters:

$$
\begin{aligned}
-\Delta^{\frac{5}{2}} u_{1}(t)= & \frac{e^{-\left(t+\frac{4}{3}\right)}\left(\left|u_{2}\right|+1\right)}{400\left(t+\frac{34}{3}\right)^{2}\left(1+\sin ^{2} u_{2} \pi\right)}+\frac{e^{-\left(t+\frac{3}{2}\right) \pi} u_{1}\left(t+\frac{3}{2}\right)}{100 e+20 \sin ^{2}\left(t+\frac{3}{2}\right) \pi} \\
-\frac{1}{2} \Delta^{\frac{7}{3}} u_{2}(t)= & \frac{e^{-\left(t+\frac{3}{2}\right)}\left(\left|u_{1}\right|+e^{-\sin ^{2}\left(t+\frac{3}{2}\right) \pi}\right)}{1000\left(e^{t+\frac{3}{2}}+10\right)^{2}\left(\left|u_{1}\right|+\cos ^{2}\left(t+\frac{3}{2}\right) \pi\right)} \\
& +\frac{\arctan \left(\sin ^{2}\left(t+\frac{4}{3}\right) \pi\right) u_{2}\left(t+\frac{4}{3}\right)}{100 \pi\left(t+\frac{13}{3}\right)^{2}}
\end{aligned}
$$

for $t \in \mathbb{N}_{0,5}$, subject to nonlocal fractional difference-sum conditions

$$
\begin{aligned}
& \Delta^{-\frac{1}{3}} u_{1}\left(-\frac{1}{6}\right)=\Delta^{\frac{1}{5}} u_{1}\left(\frac{3}{10}\right)=0, \\
& \Delta^{-\frac{1}{4}} u_{2}\left(-\frac{5}{12}\right)=\Delta^{\frac{2}{5}} u_{2}\left(-\frac{1}{15}\right)=0, \\
& u_{1}\left(\frac{15}{2}\right)=\frac{1}{2} u_{1}\left(\frac{9}{2}\right) \\
& u_{2}\left(\frac{22}{3}\right)=\frac{3}{4} u_{2}\left(\frac{13}{3}\right)
\end{aligned}
$$

Here $\alpha_{1}=\frac{5}{2}, \alpha_{2}=\frac{7}{3}, \beta_{1}=\frac{1}{3}, \beta_{2}=\frac{1}{4}, \gamma_{1}=\frac{1}{5}, \gamma_{2}=\frac{2}{5}, \lambda_{1}=1, \lambda_{2}=2, \chi_{1}=\frac{1}{2}, \chi_{2}=\frac{3}{4}, \eta_{1}=\frac{9}{2}$, $\eta_{2}=\frac{13}{3}, T=5$, and for $t_{1} \in \mathbb{N}_{-\frac{1}{2}, \frac{15}{2}}$ and $t_{2} \in \mathbb{N}_{-\frac{2}{3}, \frac{22}{3}}$,

$$
\begin{aligned}
& F_{1}\left[t_{1}, t_{2}, u_{1}, u_{2}\right]=\frac{e^{-t_{2}}\left(\left|u_{2}\right|+1\right)}{400\left(t_{2}+10\right)^{2}\left(1+\sin ^{2} u_{2} \pi\right)}+\frac{e^{-t_{1} \pi} u_{1}\left(t_{1}\right)}{100 e+20 \sin ^{2} t_{1} \pi}, \\
& F_{2}\left[t_{1}, t_{2}, u_{1}, u_{2}\right]=\frac{e^{-t_{1}}\left(\left|u_{1}\right|+e^{-\sin ^{2} t_{1} \pi}\right)}{1000\left(e^{t_{1}}+10\right)^{2}\left(\left|u_{1}\right|+\cos ^{2} t_{1} \pi\right)}+\frac{\arctan \left(\sin ^{2} t_{2} \pi\right) u_{2}\left(t_{2}\right)}{100 \pi\left(t_{2}+3\right)^{2}} .
\end{aligned}
$$

We get that

$$
\begin{aligned}
& \left|F_{1}\left[t_{1}, t_{2}, u_{1}, u_{2}\right]-F_{1}\left[t_{1}, t_{2}, v_{1}, v_{2}\right]\right| \\
& \quad \leq \frac{e^{-t_{2}}}{400\left(t_{2}+10\right)^{2}}\left|\frac{\left|u_{2}\right|+1}{1+\sin ^{2} u_{2} \pi}-\frac{\left|v_{2}\right|+1}{1+\sin ^{2} v_{2} \pi}\right|+\frac{e^{-t_{1} \pi}}{100 e+20 \sin ^{2} t_{1} \pi}\left|\Delta^{\frac{1}{3}} u_{1}-\Delta^{\frac{1}{3}} v_{1}\right|
\end{aligned}
$$




$$
\begin{aligned}
& \quad \leq \frac{1}{45,511}\left|u_{2}-v_{2}\right|+\frac{1}{100 e}\left|u_{1}-v_{1}\right|, \\
& \left|F_{2}\left[t_{1}, t_{2}, u_{1}, u_{2}\right]-F_{2}\left[t_{1}, t_{2}, v_{1}, v_{2}\right]\right| \\
& \left.\quad \leq \frac{e^{-t_{1}}}{1000\left(e^{t_{1}}+10\right)^{2}}\left|\frac{\left|u_{1}\right|}{1+\left|u_{1}\right|}-\frac{\left|v_{1}\right|}{1+\left|v_{1}\right|}\right|+\frac{\arctan (1)}{100 \pi\left(t_{2}+3\right)^{2}} \mid u_{2}-v_{2}\right) \mid \\
& \quad \leq \frac{1}{12,100}\left|u_{1}-v_{1}\right|+\frac{9}{19,600}\left|u_{2}-v_{2}\right| .
\end{aligned}
$$

So, (3.3) holds with $M_{1}=0.00002197, M_{2}=0.00008264, N_{1}=0.00368$, and $N_{2}=0.000459$. Finally, we find that

$$
\begin{aligned}
& L_{1}=\max \left\{M_{1}, N_{1}\right\}=0.00368, \quad L_{2}=\max \left\{M_{2}, N_{2}\right\}=0.000459 \quad \text { and } \\
& \Omega_{1}=75.4559, \quad \Omega_{2}=136.2638 .
\end{aligned}
$$

Therefore we obtain

$$
L_{1} \Omega_{1}+L_{2} \Omega_{2}=0.3402<1 .
$$

Hence, by Theorem 3.1, problem (5.1)-(5.2) has a unique solution.

Example 2 Consider the following system of fractional difference equations with parameters:

$$
\begin{aligned}
& -\Delta^{\frac{5}{2}} u_{1}(t)=\left[u_{1}\left(t+\frac{3}{2}\right)+u_{1}\left(t+\frac{4}{3}\right)\right]^{6}, \\
& -\frac{1}{2} \Delta^{\frac{7}{3}} u_{2}(t)=\left[u_{1}\left(t+\frac{3}{2}\right)+u_{1}\left(t+\frac{4}{3}\right)\right]^{4}, \quad t \in \mathbb{N}_{0,5}
\end{aligned}
$$

for $t \in \mathbb{N}_{0,5}$, subject to nonlocal fractional difference-sum conditions (5.2).

For all $t_{1} \in \mathbb{N}_{-\frac{1}{2}, \frac{15}{2}}, t_{2} \in \mathbb{N}_{-\frac{2}{3}, \frac{22}{3}}, u_{1}, u_{2}>0$, we have $F_{1}\left[t_{1}, t_{2}, u_{1} u_{2}\right]=\left[u_{1}\left(t_{1}\right)+u_{1}\left(t_{2}\right)\right]^{6}>0$ and $F_{2}\left[t_{1}, t_{2}, u_{1} u_{2}\right]=\left[u_{1}\left(t_{1}\right)+u_{1}\left(t_{2}\right)\right]^{4}>0$. We find that

$$
\begin{aligned}
& 0<4.3619=\chi_{1} \eta \frac{\alpha_{1}-1}{1}<\left(T+\alpha_{1}\right) \frac{\alpha_{1}-1}{2}=19.4922, \\
& 0<5.0162=\chi_{2} \eta_{2}^{\frac{\alpha_{2}-1}{2}}<\left(T+\alpha_{2}\right) \frac{\alpha_{2}-1}{=}=13.8057 .
\end{aligned}
$$

By direct calculation we have $F_{1}^{0}=F_{2}^{0}=0$ and $F_{1}^{\infty}=F_{2}^{\infty}=\infty$. Then, by Theorem 4.1(a), problem (5.3) and (5.2) has at least one positive solution.

Example 3 Consider the following system of fractional difference equations with parameters:

$$
\begin{aligned}
& -\Delta^{\frac{5}{2}} u_{1}(t)=\left[u_{1}\left(t+\frac{3}{2}\right)+u_{1}\left(t+\frac{4}{3}\right)\right]^{\frac{1}{3}}, \\
& -\frac{1}{2} \Delta^{\frac{7}{3}} u_{2}(t)=\left[u_{1}\left(t+\frac{3}{2}\right)+u_{1}\left(t+\frac{4}{3}\right)\right]^{\frac{1}{4}}, \quad t \in \mathbb{N}_{0,5},
\end{aligned}
$$

for $t \in \mathbb{N}_{0,5}$, subject to nonlocal fractional difference-sum conditions (5.2). 
For all $t_{1} \in \mathbb{N}_{-\frac{1}{2}, \frac{15}{2}}, t_{2} \in \mathbb{N}_{-\frac{2}{3}, \frac{22}{3}}, u_{1}, u_{2}>0$, we have $F_{1}\left[t_{1}, t_{2}, u_{1} u_{2}\right]=\left[u_{1}\left(t_{1}\right)+u_{1}\left(t_{2}\right)\right]^{\frac{1}{3}}>0$ and $F_{2}\left[t_{1}, t_{2}, u_{1} u_{2}\right]=\left[u_{1}\left(t_{1}\right)+u_{1}\left(t_{2}\right)\right]^{\frac{1}{4}}>0$.

By the same argument as in Example 2 we have that $\chi_{i} \eta_{i}^{\frac{\alpha_{i}-1}{1}}<\left(T+\alpha_{i}\right) \frac{\alpha_{i}-1}{2}, i=1,2$.

By direct calculation we have $F_{1}^{0}=F_{2}^{0}=\infty$ and $F_{1}^{\infty}=F_{2}^{\infty}=0$. Then, by Theorem 4.1(b), problem (5.4) and (5.2) has at least one positive solution.

\section{Acknowledgements}

This research was supported by Kasetsart University.

\section{Funding}

This research was funded by King Mongkut's University of Technology North Bangkok, contract no. KMUTNB-61-KNOW-028.

Availability of data and materials

Not applicable.

\section{Ethics approval and consent to participate}

Not applicable.

\section{Competing interests}

The authors declare that they have no competing interests.

\section{Consent for publication}

Not applicable.

\section{Authors' contributions}

All authors read and approved the final manuscript.

\section{Author details}

${ }^{1}$ Department of Mathematics, Faculty of Science, Kasetsart University, Bangkok 10900, Thailand. ${ }^{2}$ Department of Mathematics, Faculty of Applied Science, King Mongkut's University of Technology North Bangkok, Bangkok 10800, Thailand. ${ }^{3}$ Mathematics Department, Faculty of Science and Technology, Suan Dusit University, Bangkok 10300, Thailand.

\section{Publisher's Note}

Springer Nature remains neutral with regard to jurisdictional claims in published maps and institutional affiliations.

Received: 20 April 2020 Accepted: 17 August 2020 Published online: 27 August 2020

\section{References}

1. Wu, G.C., Baleanu, D.: Discrete fractional logistic map and its chaos. Nonlinear Dyn. 75, 283-287 (2014)

2. Wu, G.C., Baleanu, D.: Chaos synchronization of the discrete fractional logistic map. Signal Process. 102, 96-99 (2014)

3. Wu, G.C., Baleanu, D., Xie, H.P., Chen, F.L.: Chaos synchronization of fractional chaotic maps based on stability results. Phys. A. 460, 374-383 (2016)

4. Goodrich, C.S., Peterson, A.C.: Discrete Fractional Calculus. Springer, New York (2015)

5. Atici, F.M., Eloe, P.W.: A transform method in discrete fractional calculus. Int. J. Differ. Equ. 2(2), 165-176 (2007)

6. Atici, F.M., Eloe, P.W.: Two-point boundary value problems for finite fractional difference equations. J. Differ. Equ. Appl. $17,445-456(2011)$

7. Abdeljawad, T.: On Riemann and Caputo fractional differences. Comput. Math. Appl. 62(3), 1602-1611 (2011)

8. Jia, B., Erbe, L., Peterson, A.: Two monotonicity results for nabla and delta fractional differences. Arch. Math. 104, 589-597 (2015)

9. Jia, B., Erbe, L., Peterson, A.: Convexity for nabla and delta fractional differences. J. Differ. Equ. Appl. 21, 360-373 (2015)

10. Ferreira, R.A.C.: Existence and uniqueness of solution to some discrete fractional boundary value problems of order less than one. J. Differ. Equ. Appl. 19, 712-718 (2013)

11. Ferreira, R.A.C., Goodrich, C.S.: Positive solution for a discrete fractional periodic boundary value problem. Dyn. Contin. Discrete Impuls. Syst., Ser. A Math. Anal. 19, 545-557 (2012)

12. Goodrich, C.S.: Existence and uniqueness of solutions to a fractional difference equation with nonlocal conditions. Comput. Math. Appl. 61, 191-202 (2011)

13. Goodrich, C.S.: On a discrete fractional three-point boundary value problem. J. Differ. Equ. Appl. 18, $397-415$ (2012)

14. Erbe, L., Goodrich, C.S., Jia, B., Peterson, A.: Survey of the qualitative properties of fractional difference operators: monotonicity, convexity, and asymptotic behavior of solutions. Adv. Differ. Equ. 2016, 43, 31 pages (2016)

15. Chen, Y., Tang, X.: Three difference between a class of discrete fractional and integer order boundary value problems. Commun. Nonlinear Sci. Numer. Simul. 19(12), 4057-4067 (2014)

16. LV, W., Feng, J.: Nonlinear discrete fractional mixed type sum-difference equation boundary value problems in Banach spaces. Adv. Differ. Equ. 2014, 184, 12 pages (2014)

17. Weidong, Lv.: Existence of solutions for discrete fractional boundary value problems with a $p$-Laplacian operator. Adv. Differ. Equ. 2012, 163 (2012) 
18. Kang, S.G., Li, Y., Chen, H.Q.: Positive solutions to boundary value problems of fractional difference equations with nonlocal conditions. Adv. Differ. Equ. 2014, 7, 12 pages (2014)

19. Dong, W., Xu, J., Regan, D.O.: Solutions for a fractional difference boundary value problem. Adv. Differ. Equ. 2013, 319, 12 pages (2013)

20. Holm, M.: Sum and difference compositions in discrete fractional calculus. CUBO 13(3), 153-184 (2011)

21. Sitthiwirattham, T., Tariboon, J., Ntouyas, S.K.: Existence results for fractional difference equations with three-point fractional sum boundary conditions. Discrete Dyn. Nat. Soc. 2013, Article ID 104276, 9 pages (2013)

22. Sitthiwirattham, T., Tariboon, J., Ntouyas, S.K.: Boundary value problems for fractional difference equations with three-point fractional sum boundary conditions. Adv. Differ. Equ. 2013, 296, 13 pages (2013)

23. Sitthiwirattham, T.: Existence and uniqueness of solutions of sequential nonlinear fractional difference equations with three-point fractional sum boundary conditions. Math. Methods Appl. Sci. 38, 2809-2815 (2015)

24. Sitthiwirattham, T: Boundary value problem for $p$-Laplacian Caputo fractional difference equations with fractional sum boundary conditions. Math. Methods Appl. Sci. 39(6), 1522-1534 (2016)

25. Chasreechai, S., Kiataramkul, C., Sitthiwirattham, T.: On nonlinear fractional sum-difference equations via fractional sum boundary conditions involving different orders. Math. Probl. Eng. 2015, Article ID 519072, 9 pages (2015)

26. Reunsumrit, J., Sitthiwirattham, T.: Positive solutions of three-point fractional sum boundary value problem for Caputo fractional difference equations via an argument with a shift. Positivity 20(4), 861-876 (2016)

27. Reunsumrit, J., Sitthiwirattham, T.: On positive solutions to fractional sum boundary value problems for nonlinear fractional difference equations. Math. Methods Appl. Sci. 39(10), 2737-2751 (2016)

28. Soontharanon, J., Jasthitikulchai, N., Sitthiwirattham, T.: Nonlocal fractional sum boundary value problems for mixed types of Riemann-Liouville and Caputo fractional difference equations. Dyn. Syst. Appl. 25, 409-414 (2016)

29. Laoprasittichok, S., Sitthiwirattham, T:: On a fractional difference-sum boundary value problems for fractional difference equations involving sequential fractional differences via different orders. J. Comput. Anal. Appl. 23(6), 1097-1111 (2017)

30. Kaewwisetkul, B., Sitthiwirattham, T.: On nonlocal fractional sum-difference boundary value problems for Caputo fractional functional difference equations with delay. Adv. Differ. Equ. 2017, 219, 14 pages (2017)

31. Reunsumrit, J., Sitthiwirattham, T.: A new class of four-point fractional sum boundary value problems for nonlinear sequential fractional difference equations involving shift operators. Kragujev. J. Math. 42(3), 371-387 (2018)

32. Chasreechai, S., Sitthiwirattham, T.: Existence results of initial value problems for hybrid fractional sum-difference equations. Discrete Dyn. Nat. Soc. 2018, Article ID 5268528 (2018)

33. Chasreechai, S., Sitthiwirattham, T:: On separate fractional sum-difference boundary value problems with $n$-point fractional sum-difference boundary conditions via arbitrary different fractional orders. Mathematics 2019(7), Article ID 471 (2019)

34. Pan, Y., Han, Z., Sun, S., Zhao, Y.: The existence of solutions to a system of discrete fractional boundary value problems. Abstr. Appl. Anal. 2012, Article ID 707631, 15 pages (2012)

35. Goodrich, C.S.: Existence of a positive solution to a system of discrete fractional boundary value problems. Appl. Math. Comput. 217(9), 4740-4753 (2011)

36. Dahal, R., Duncan, D., Goodrich, C.S.: Systems of semipositone discrete fractional boundary value problems. J. Differ. Equ. Appl. 20(3), 473-491 (2014)

37. Goodrich, C.S.: Systems of discrete fractional boundary value problems with nonlinearities satisfying no growth conditions. J. Differ. Equ. Appl. 21(5), 437-453 (2015)

38. Goodrich, C.S.: Coupled systems of boundary value problems with nonlocal boundary conditions. Appl. Math. Lett. $41,17-22(2015)$

39. Kunnawuttipreechachan, E., Promsakon, C., Sitthiwirattham, T.: Nonlocal fractional sum boundary value problems for a coupled system of fractional sum-difference equations. Dyn. Syst. Appl. 28(1), 73-92 (2019)

40. Promsakon, C., Chasreechai, S., Sitthiwirattham, T.: Positive solution to a coupled system of singular fractional difference equations with fractional sum boundary value conditions. Adv. Differ. Equ. 2019, Article ID 218 (2019)

41. Soontharanon, J., Chasreechai, S., Sitthiwirattham, T.: On a coupled system of fractional difference equations with nonlocal fractional sum boundary value conditions on the discrete half-line. Mathematics 2019(7), Article ID 256 (2019)

42. Siricharuanun, P., Chasreechai, S., Sitthiwirattham, T.: On a coupled system of fractional sum-difference equations with p-Laplacian operator. Adv. Differ. Equ. 2020, Article ID 361 (2020)

43. Guo, D., Lakshmikantham, V.: Nonlinear Problems in Abstract Cone. Academic Press, Orlando (1988)

44. Griffel, D.H.: Applied Functional Analysis. Ellis Horwood, Chichester (1981)

\section{Submit your manuscript to a SpringerOpen ${ }^{\circ}$ journal and benefit from:}

- Convenient online submission

- Rigorous peer review

- Open access: articles freely available online

- High visibility within the field

- Retaining the copyright to your article

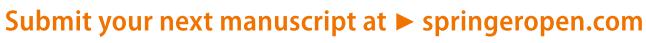

